\title{
Competition and collaboration in the miRNA science field
}

\author{
Artemiy Firsov \\ Computer Science and Computer Engineering \\ Institute of Informarics Systems \\ Novosibirsk, Russia \\ 0000-0002-7681-1032
}

\begin{abstract}
In this work we present the analysis of the characteristics of institutions interactions in the miRNA science field using the data from PubMed digital library. We identified the leaders of the field - China, USA -, characterized the interactions and described the country level features of coauthorship. We observed that the USA were leading in the publication activity until China took the lead 4 years ago. However, the USA are the main co-authorship driver in this field. We have also identified the pioneers and show, that they are the leaders of co-authorship activity. We compare the publications activity patterns on the organization level, identifying leaders. We compare the organization interaction graph with the authors interaction graph, and provide additional insights of miRNA science field evolution.
\end{abstract}

Keywords - k-mer, n-gram, dbscan, identification, mirna, timsort, kofer, digital library, co-authorship

Introduction

Many digital libraries appeared with the growth of the Internet, thus, we got an opportunity to query articles metadata, gather some statistics, etc. This includes understanding the authors/institutions activity, their interactions, and other characteristics. One can also prove that the Paretto rule for the institutions' publication activity holds true [1], or that the idea spreads from one author to another like the virus spreads from one person to the others. Having this information, we can further use it to predict the new science field creation, popularity of the particular science field. In general, it can be used in social informatics.

Now the new science field is progressing - "science of science" [2]. It is a trans-disciplinary field of science that aims to understand the evolution of ideas, choice of a research problem of particular scientist, etc. Without analyzing interactions between authors, institutions, and other, such field just cannot exist.

However, to do that one should know to which real author/institution the authors name/affiliation from the paper corresponds to. This disambiguation issue gets harder considering big datasets, such as PubMed with $3 * 10^{7}$ articles. It becomes more complicated when you consider the presence of non-affiliation data in the affiliation field, errors in the author name/affiliation or the mixed institution names for different authors. E.g. if the Authorl has "Institute of Cytology and Genetics, Novosibirsk, Russia" institution and the Author2 has "Institute of Mathematics, Novosibirsk, Russia" institution, their resulting affiliation for collaborative paper might be "Institute of Cytology and Genetics, Institute of Mathematics, Novosibirsk, Russia".

In this paper, we aim to provide the solution for Affiliation Disambiguation problem and provide the analysis of the miRNA science field using the solution.

\author{
Igor Titov \\ Laboratory of Molecular Genetic Systems \\ Institute of Cytology and Genetics \\ Novosibirsk, Russia \\ titov@bionet.nsc.ru
}

Methods and Materials

Prerequisites

The basic idea of the work is to get the groups of organizations mentions, which contain only mentions of one institution. After that we may use that information to build the co-authorship graph of organizations/countries, get static and dynamic characteristics of the science field, etc.

So on the first step we solved the clustering problem of institutions names writings. In our case, the closer the grouping is to the ground-truth grouping (i.e. one group contains all and only affiliations that refer to the same institution), the better the clustering is. For that sake, we have gathered the labeled the Novosibirsk miRNA dataset mentioned below.

\section{Dataset}

To conduct experiments, we have gathered two datasets from PubMed digital library using MEDLINE file format. First one is the Novosibirsk dataset, that consists of the preprocessed affiliations of the Novosibirsk institutions. We labeled this dataset to have the ground-truth affiliation clustering to further use it for clustering algorithm hyperparameters fine-tuning. You can find the additional information on the Novosibirsk dataset, and how we used it to fine-tune our algorithm, in our previous paper dedicated to the inter-country statistics of the miRNA science field [3].

Second one is the miRNA dataset gathered with the $m i R N A$ search query and its forms ( $m i-R N A, m i R N A S$, etc) on the PubMed website over Title and Abstract fields. The data we use was gathered on $18^{\text {th }}$ of November, 2019.

\section{Clustering and similarity}

After the pre-processing stage, where we remove nonaffiliation data and split the mixed affiliations, the clustering stage is performed. We tried different techniques for the clustering - K-Means and DBSCAN [4] - and different popular similarity functions applied to the preprocessed affiliations strings - Levenshtein, Jaccard, SmithWaterman. We also tried the KOFER algorithm [3] to find similarity between affiliations.

We found out that KOFER algorithm along with Dice [5] distance metric gives better results on the labeled Novosibirsk dataset; thus, we used that approach on the full miRNA dataset.

\section{Results}

We present the results for country level co-authorship in the miRNA field, as well as the organization level statistics, i.e. pioneer analysis, leaders, etc. Using the KOFER algorithm we were able to cluster the miRNA affiliations data. From the 530,982 affiliations we got 34,081 clusters. i.e. institutions. 


\section{PubMed statistics}

We got the cumulative per year number of publications on the PubMed digital library. We found out that the total number of publications added yearly to PubMed grows exponentially:

$$
n(t)=e^{0.0492 * x+13.1902}
$$

where $\mathrm{t}-0,1,2 \ldots$ and 0 corresponds to the starting year of publication activity within the miRNA field, i.e. 2001

miRNA statisics

The growth of the publications per year within the miRNA field, however, is reaching its peak, and can further be estimated using the models with saturation, such as lognormal and log-logistic functions.

\section{Countries interactions}

146 countries have publications in the miRNA science field. To see how countries interact, we have built the graph, leaving only the major countries in this filed, i.e. having more than 500 publications.

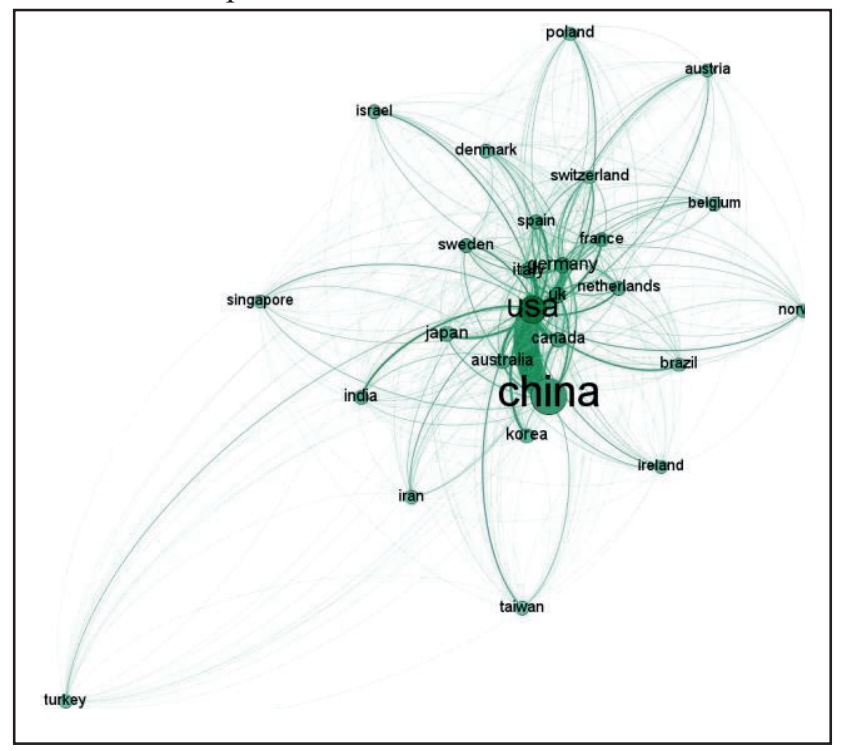

From this graph, it is clear that the USA and China are the leaders in this field, actively publishing with each other. This graph is also almost full, however, some of the countries have never published together, such as Iran-Israel, Iran-Brazil, Turkey-Belgium.

Additionally, analyzing the plots of publications per year, we noticed that the USA had the rapid start in this field, reaching 100 publications in 2004. However, the growth started to reduce over time, whereas China had higher growth, which led to China becoming the new leader in 2013. The USA on the other side remained the major driver of the international publications, and has more than others publications with China, Italy, UK, Canada, Germany, Korea, Japan.

\section{Organization interactions}

During the 2001-2019 period, there were 88,715 publications, 12,857 of which were international. The field until some time did not have much joint publications, however things changed in 2013. That year USA and China started actively publish together.

The interesting thing that the pioneers (the organizations, which published during the period 2001 2003) within this field have higher international publications rate than the overall rate -6128 publications were published with pioneers, 2640 of which were international. Moreover, first four most active international publishers are the pioneers - University of California, Harvard Medical School, University of Texas MD Anderson Cancer Centre, Chinese Academy of Sciences.

We observer that out of 34,081 institutions, there are only 1,655 organizations, for which the number of their mentions is higher than 50 (1\% of maximum mentions of the leader organization - Central South University, China). All 46 pioneers are in this main group.

\section{Conclusion}

In this work we have managed to cluster the miRNA science field affiliations data using the KOFER algorithm.

Using the clustering results, we were able to get properties of country level interactions, see that China is currently the leading country in this field, however the USA is the biggest driver of international publications.

The pioneers, such as University of California, Harvard Medical School, University of Texas MD Anderson Cancer Centre, Chinese Academy of Sciences, play big role within this field, leading the international activities. The current organization leader of the miRNA science field is Central South University, China.

\section{REFERENCES}

[1] I. I. Titov, A. A. Blinov, "Research of the structure and evolution of networks of scientific cooperation on the basis of analysis of Novosibirsk publications on the field of biology and medicine," Vavilov Journal of Genetics and Breeding, vol. 18, 2014.

[2] S. Fortunato, C. Bergstrom, K. Borner, J. Evans, D. Helbing, S. Milojevic, A. Petersen, F. Radicchi, R. Sinatra, B. Uzzi, A. Vespignani, L. Waltman, D. Wang, A.-L. Barabasi, "Science of science", Science, vol. 359, 2018

[3] A Firsov, I. Titov, "Inter-country Competition and Collaboration in the miRNA Science Field", Perspectives of System Informatics, pp. 26-40, 2019

[4] M. Ester, H.-P. Kriegel, J. Sander, X. Xu, "A Density-Based Algorithm for Discovering Clusters a Density-Based Algorithm for Discovering Clusters in Large Spatial Databases with Noise", Proceedings of the Second International Conference on Knowledge Discovery and Data Mining, pp. 226-231, 1996

[5] L. R. Dice, "Measures of the Amount of Ecologic Association Between Species", vol. 26, pp. 297-302, 1945

[6] Y. Yorozu, M. Hirano, K. Oka, and Y. Tagawa, "Electron spectroscopy studies on magneto-optical media and plastic substrate interface," IEEE Transl. J. Magn. Japan, vol. 2, pp. 740-741, August 1987 [Digests 9th Annual Conf. Magnetics Japan, p. 301, 1982].

[7] M. Young, The Technical Writer's Handbook. Mill Valley, CA: University Science, 1989. 